\title{
Mellin Transform Method for European Option Pricing with Hull-White Stochastic Interest Rate
}

\author{
Ji-Hun Yoon \\ Department of Mathematical Science, Seoul National University, Seoul 151-747, Republic of Korea \\ Correspondence should be addressed to Ji-Hun Yoon; ysscisnu@snu.ac.kr
}

Received 5 May 2014; Accepted 9 September 2014; Published 16 October 2014

Academic Editor: Li Ma

Copyright (C) 2014 Ji-Hun Yoon. This is an open access article distributed under the Creative Commons Attribution License, which permits unrestricted use, distribution, and reproduction in any medium, provided the original work is properly cited.

Even though interest rates fluctuate randomly in the marketplace, many option-pricing models do not fully consider their stochastic nature owing to their generally limited impact on option prices. However, stochastic dynamics in stochastic interest rates may have a significant impact on option prices as we take account of issues of maturity, hedging, or stochastic volatility. In this paper, we derive a closed form solution for European options in Black-Scholes model with stochastic interest rate using Mellin transform techniques.

\section{Introduction}

In practice, random fluctuations of interest rate over time have a significant contribution to the change of an option price. Based on this observation, some work has been reported on the price formula of European options with stochastic interest rate. Most of all, Merton [1], Rabinovitch [2], and Amin and Jarrow [3] have proposed the formula of closed form European option pricing under the Gaussian interest rate by using relatively simple algebra. This method is also discussed in detail by Kim [4]. Also, Fang [5] derived an exact pricing formula for European option under stochastic interest rate by applying martingale method. However, the closed formula for the prices of options has been studied usually by utilizing probabilistic techniques as the papers stated above. In this paper, we use analytic methods based on Mellin transforms as a better way to compute the option prices.

The Mellin transform is defined as an integral transform that may be considered as the multiplicative version of the two-sided Laplace transform. Many papers have shown that the Mellin transform technique would help us resolve the complexity of the calculation compared to the probabilistic approach. Panini and Srivastav [6] studied the pricing formula of a European vanilla option and a basket option using Mellin transforms. Panini and Srivastav [7] found also the pricing of perpetual American options with Mellin transforms. Frontczak and Schöbel [8] used Mellin transforms to value American call options on dividend-paying stocks. Also, Elshegmani and Ahmed [9] derived analytical solution for an arithmetic Asian option using Mellin transforms.

This paper is organized as follows. In Section 2, we formulate a European vanilla option with Hull-White interest rate and obtain a partial differential equation (PDE) for European call option under the stochastic interest rate. In Section 3, we apply Mellin transforms to derive a closed form solution of the option price with respect to a European call option and a European put option. In Section 4, we have concluding remarks.

\section{Model Formulation}

Let $S_{t}$ be the value of the asset (stock) underlying the option, let $\mu_{t}$ be the drift rate of the stock, and let $\sigma$ be the volatility of the underlying asset. Then, the dynamics of $S_{t}$ is given by the SDE $d S_{t}=\mu_{t} S_{t} d t+\sigma S_{t} d W_{t}$, where $W_{t}$ is the standard Brownian motion. Under a risk-neutral probability measure, the above given model is transformed into the SDEs:

$$
\begin{aligned}
& d S_{t}=r_{t} S_{t} d t+\sigma S_{t} d W_{t}^{*}, \\
& d r_{t}=\left(b(t)-a r_{t}\right) d t+\check{\sigma} d W_{t}^{(r)},
\end{aligned}
$$


where $W_{t}^{*}$ represents the standard Brownian motion under a risk-neutral world satisfying the following relation:

$$
W_{t}^{*}=W_{t}+\int_{0}^{t} \frac{\mu_{s}-r_{s}}{\sigma}
$$

and the correlation of $W_{t}^{*}$ and $W_{t}^{(r)}$ is expressed by $d\left\langle W_{t}^{*}, W_{t}^{(r)}\right\rangle_{t}=\rho_{s r} d t$, where $-1 \leq \rho_{s r} \leq 1$. Also, $r_{t}$ is the Hull-White interest rate model, $a$ is mean reversion rate of interest, $\check{\sigma}$ is volatility of interest rate, and $b(t)$ is average direction of interest rate movement. Using notation $E^{*}\{\cdot\}$ as the expectation with respect to the risk-neutral measure, we have the no-arbitrage price of a European option with a payoff function $h(x)$ given by

$$
P(t, s, r)=E^{*}\left\{\exp \left(-\int_{t}^{T} r_{t^{*}} d t^{*}\right) h\left(S_{T}\right) \mid S_{t}=s, r_{t}=r\right\} .
$$

From Feynman-Kac formula (cf. [10]), the solution of $P(t, s, r)$ satisfies the following PDE:

$$
\begin{gathered}
\widehat{\mathscr{L}} P(t, s, r)=0, \\
\widehat{\mathscr{L}}=\frac{\partial}{\partial t}+\frac{1}{2} \sigma^{2} s^{2} \frac{\partial^{2}}{\partial s^{2}}+r\left(s \frac{\partial}{\partial s}-I\right) \\
+\rho_{s r} \sigma \check{\sigma} s \frac{\partial^{2}}{\partial s \partial r}+(b(t)-a r) \frac{\partial}{\partial r}+\frac{1}{2} \check{\sigma}^{2} \frac{\partial^{2}}{\partial r^{2}},
\end{gathered}
$$

where $P(T, s, r)=h(s)$ is the terminal condition and $I$ is the identity operator.

2.1. A Review of Bond Pricing Formula. Under the Hull-White interest rate model for the short rate, $r$, given by $d r_{t}=(b(t)-$ $\left.a r_{t}\right) d t+\check{\sigma} d W_{t}^{(r)}$, the no-arbitrage price at time $t$ of a zerocoupon bond maturing at time $T$ is as follows:

$$
B(t, r ; T)=E^{*}\left\{\exp \left(-\int_{t}^{T} r_{t^{*}} d t^{*}\right) \mid r_{t}=r\right\}
$$

with the final condition $B(T, r ; T)=1$.

Then, by using Feynman-Kac formula (cf. [10]), the solution of $B(t, r ; T)$ satisfies the following equation:

$$
\begin{gathered}
\frac{\partial B}{\partial t}+\frac{1}{2} \check{\sigma}^{2} \frac{\partial^{2} B}{\partial r^{2}}+(b(t)-a r) \frac{\partial B}{\partial r}-r B=0, \\
B(T, r ; T)=1,
\end{gathered}
$$

and by writing the form of the solution as $B(T-\tau, r ; T)=$ $A(\tau) e^{-D(\tau) r}$, where $\tau=T-t, A(\tau)$ and $D(\tau)$ are given by

$$
\begin{gathered}
A(\tau)=\exp \left(\frac{\check{\sigma}^{2}}{2 a^{2}}\left(\tau+\frac{2}{a}\left(e^{-a \tau}-1\right)-\frac{1}{2 a}\left(e^{-2 a \tau}-1\right)\right)\right. \\
\left.-\int_{0}^{\tau} b\left(T-\tau^{*}\right) D\left(\tau^{*}\right) d \tau^{*}\right), \\
D(\tau)=\frac{1-e^{-a \tau}}{a} .
\end{gathered}
$$

In Section 3, we will define $\left(\check{\sigma}^{2} / 2 a^{2}\right)\left(\tau+(2 / a)\left(e^{-a \tau}-1\right)-\right.$ $\left.\left.(1 / 2 a)\left(e^{-2 a \tau}-1\right)\right), \int_{0}^{\tau} b\left(T-\tau^{*}\right) D\left(\tau^{*}\right) d \tau^{*}\right)$, and $D(\tau)$ as $M_{1}(\tau)$, $M_{2}(\tau)$, and $G(\tau)$, respectively.

2.2. A Review of the Mellin Transforms. To derive a closed solution of $P(t, s, r)$, we use the Mellin transform. For a locally Lebesgue integrable function $f(x), x \in \mathbb{R}^{+}$, the Mellin transform $\mathscr{M}(f(x), w), w \in \mathbb{C}$, is defined by

$$
\mathscr{M}(f(x), w):=\widehat{f}(w)=\int_{0}^{\infty} f(x) x^{w-1} d x,
$$

and if $a<\operatorname{Re}(w)<b$ and $c$ such that $a<c<b$ exists, the inverse of the Mellin transform is expressed by

$$
f(x)=\mathscr{M}^{-1}(\widehat{f}(w))=\frac{1}{2 \pi i} \int_{c-i \infty}^{c+i \infty} \widehat{f}(w) x^{-w} d w .
$$

\section{The Derivation of the Formula of European Option Price}

3.1. The Case of Call Option. In this section, we derive the formula of European call option with the Hull-White interest rate using the Mellin transform. However, since the European call option has the payoff function $P(T, s, r)=h(s)=$ $(s-K)^{+}$, the Mellin transform of the payoff function does not exist. Therefore, a somewhat modified form of $h(s)=(s-K)^{+}$ is needed to guarantee the existence of the integral, and we define the sequence of the payoff function $h_{n}(s)$ such that $\lim _{n \rightarrow \infty} h_{n}(s)=h(s)$ as follows:

$$
h_{n}(S)= \begin{cases}S_{T}-K, & \text { if } K<S_{T}<n \\ 0, & \text { if } S_{T}<K .\end{cases}
$$

If we define the call option price with the payoff function $h_{n}(s)=P_{n}(T, s, r)$ as

$$
P_{n}(t, s, r)=E^{*}\left\{\exp \left(-\int_{t}^{T} r_{t^{*}} d t^{*}\right) h_{n}\left(S_{T}\right) \mid S_{t}=s, r_{t}=r\right\},
$$

$P_{n}(t, s, r)$ satisfies the PDE given by (4). Then, if we find the solution $P_{n}(t, s, r)$ by using the Mellin transform, we can obtain the formula of the option price $P(t, s, r)$ from $P(t, s, r)=\lim _{n \rightarrow \infty} P_{n}(t, s, r)$.

If we define $\widehat{p}_{n}(t, w, r)$ as the Mellin transform of $P_{n}(t, s, r)$, then the inverse of the Mellin transform is given by

$$
P_{n}(t, s, r)=\frac{1}{2 \pi i} \int_{c-i \infty}^{c+i \infty} \widehat{p}_{n}(t, w, r) s^{-w} d w .
$$

By substituting (12) into the PDE $\widehat{\mathscr{L}} P_{n}=0$ mentioned above, the PDE is transformed by

$$
\begin{aligned}
& -\frac{\partial \widehat{p}_{n}}{\partial \tau}+\left(\frac{1}{2} \sigma^{2} w(w+1)-r w-r\right) \widehat{p}_{n} \\
& \quad+\left(b(T-\tau)-a r-\rho_{s r} \sigma \check{\sigma} w\right) \frac{\partial \widehat{p}_{n}}{\partial r}+\frac{1}{2} \check{\sigma}^{2} \frac{\partial^{2} \widehat{p}_{n}}{\partial r^{2}}=0,
\end{aligned}
$$


where $\tau=T-t$ and the terminal condition is given by $\widehat{p}_{n}(0, w, r)=\int_{0}^{\infty} P_{n}(0, s, r) s^{w-1} d s=\int_{K}^{n}(s-K) s^{w-1} d s=$ $n^{w}(n /(w+1)-K / w)+K^{w+1} / w(w+1)$.

To simplify PDE (13), let us assume $\widehat{p}_{n}(\tau, w, r)$ can be expressed by the form

$$
\widehat{p}_{n}(\tau, w, r)=\exp \left\{\frac{1}{2} \sigma^{2} w(w+1) \tau\right\} \widehat{g}_{n}(\tau, w, r),
$$

and then $\widehat{g}_{n}$ must satisfy

$$
\begin{gathered}
-\frac{\partial \widehat{g}_{n}}{\partial \tau}+\left(-a r+\left(b(T-\tau)-\rho_{s r} \sigma \check{\sigma} w\right)\right) \frac{\partial \widehat{g}_{n}}{\partial r} \\
+\frac{1}{2} \check{\sigma}^{2} \frac{\partial^{2} \widehat{g}_{n}}{\partial r^{2}}-r(w+1) \widehat{g}_{n}=0
\end{gathered}
$$

with the final condition $\widehat{g}_{n}(0, w, r)=\widehat{p}_{n}(0, w, r)=n^{w}(n /(w+$ 1) $-K / w)+K^{w+1} / w(w+1)$.

Now, to solve $\operatorname{PDE}(15)$, we set $\hat{g}_{n}(\tau, w, r)=$ $E_{n}(w) H(\tau, w) e^{-G(\tau)(w+1) r}$. Here, $\widehat{g}_{n}(0, w, r)=E_{n}(w)$ and by substituting this functional form of the solution into PDE (15), we have two ordinary differential equations (ODEs) with respect to $H(\tau, w)$ and $G(\tau)$ as follows:

$$
\begin{gathered}
-H_{\tau}-\left(\left(b(T-\tau)-\rho_{s r} \sigma \check{\sigma} w\right) G(\tau)(w+1)\right. \\
\left.-\frac{1}{2} \check{\sigma}^{2} G^{2}(\tau)(w+1)^{2}\right) H=0, \\
G^{\prime}(\tau)+a G(\tau)-1=0
\end{gathered}
$$

with $H(0, w)=1$ and $G(0)=0$. ODEs (16) yield

$$
\begin{gathered}
H(\tau, w)=\exp \left\{\int_{0}^{\tau} L\left(\tau^{*}, w\right) d \tau^{*}\right\}, \\
G(\tau)=\frac{1-e^{-a \tau}}{a},
\end{gathered}
$$

where $L(\tau, w)=(1 / 2) \check{\sigma}^{2} G^{2}(\tau)(w+1)^{2}-(b(T-\tau)-$ $\left.\rho_{s r} \sigma \check{\sigma} w\right) G(\tau)(w+1)$.

Therefore, from the solution of $\hat{g}_{n}(\tau, w, r)$ and (14), we obtain the solution of $\widehat{p}_{n}(\tau, w, r)$ as follows:

$$
\begin{aligned}
& \widehat{p}_{n}(\tau, w, r) \\
& =E_{n}(w) \exp \left\{M_{1}(\tau)(w+1)^{2}+\frac{1}{2} \sigma^{2} w(w+1) \tau\right. \\
& +\eta w(w+1)(\tau-G(\tau))-M_{2}(\tau)(w+1) \\
& \quad-G(\tau) r\},
\end{aligned}
$$

where

$$
\begin{gathered}
M_{1}(\tau)=\frac{\check{\sigma}^{2}}{2 a^{2}}\left(\tau+\frac{2}{a}\left(e^{-a \tau}-1\right)-\frac{1}{2 a}\left(e^{-2 a \tau}-1\right)\right) \\
M_{2}(\tau)=\int_{0}^{\tau} G\left(\tau^{*}\right) b\left(T-\tau^{*}\right) d \tau^{*}, \\
\eta=\frac{\rho_{s r} \sigma \check{\sigma}}{a} .
\end{gathered}
$$

From the definition of $M_{1}(\tau), M_{2}(\tau)$, and $G(\tau)$, the bond price stated in Section 2.1 is

$$
B(T-\tau, r ; T)=\exp \left\{M_{1}(\tau)-M_{2}(\tau)-G(\tau) r\right\} .
$$

Finally, we have the price of the European call option:

$$
\begin{aligned}
& P_{n}(t, w, r) \\
& =\frac{1}{(2 \pi i)} \\
& \quad \times \int_{\mathcal{c}-i \infty}^{c+i \infty} E_{n}(w) \exp \left\{M_{1}(\tau)(w+1)^{2}+\frac{1}{2} \sigma^{2} w(w+1) \tau\right. \\
& +\eta w(w+1)(\tau-G(\tau)) \\
& \left.\quad-M_{2}(\tau)(w+1)-G(\tau) r\right\}
\end{aligned}
$$

$$
\times s^{-w} d w
$$

and to compute the above integral (21), we define the following equation:

$$
\gamma(s)=\frac{1}{(2 \pi i)} \int_{c-i \infty}^{c+i \infty} e^{\varphi(\tau, w, r)} s^{-w} d w
$$

where $\varphi(\tau, w, r)=\left\{M_{1}(\tau)(w+1)^{2}+(1 / 2) \sigma^{2} w(w+1) \tau+\eta w(w+\right.$ $\left.1)(\tau-G(\tau))-M_{2}(\tau)(w+1)-G(\tau) r\right\}$. Then $\gamma(s)$ leads to the following equation:

$\gamma(s)$

$$
\begin{array}{r}
\frac{1}{(2 \pi i)} \int_{c-i \infty}^{c+i \infty} \exp \left\{\left(\frac{1}{2} \sigma^{2} \tau+M_{1}(\tau)+\eta(\tau-G(\tau))\right) w^{2}\right. \\
+\left(\frac{1}{2} \sigma^{2} \tau+2 M_{1}(\tau)-M_{2}(\tau)\right. \\
+\eta(\tau+G(\tau))-G(\tau) r) w
\end{array}
$$

$$
\begin{array}{r}
\left.+M_{1}(\tau)-M_{2}(\tau)-G(\tau) r\right\} s^{-w} d w \\
=\exp \left\{-\frac{1}{4} \frac{\left((1 / 2) \sigma^{2} \tau+\eta(\tau-G(\tau))+M_{2}(\tau)+G(\tau) r\right)^{2}}{(1 / 2) \sigma^{2} \tau+M_{1}(\tau)+\eta(\tau-G(\tau))}\right\} \\
\cdot \frac{1}{(2 \pi i)}
\end{array}
$$




$$
\begin{array}{r}
\times \int_{c-i \infty}^{c+i \infty} \exp \left\{\left(\frac{1}{2} \sigma^{2} \tau+M_{1}(\tau)+\eta(\tau-G(\tau))\right)\right. \\
\times\left\{w+\frac{1}{2}\left(1+\left(M_{1}(\tau)-M_{2}(\tau)-G(\tau) r\right)\right.\right. \\
\times\left(\frac{1}{2} \sigma^{2} \tau+M_{1}(\tau)\right. \\
\left.\left.\left.+\eta(\tau-G(\tau)))^{-1}\right)\right\}^{2}\right\}
\end{array}
$$$$
\times s^{-w} d w
$$

To compute the integral of (23), we use the following lemma.

Lemma 1. Let $\alpha$ and $\beta$ be complex numbers satisfying $\operatorname{Re}(\alpha) \geq$ 0 . Then,

$$
\frac{1}{2 \pi i} \int_{c-i \infty}^{c+i \infty} e^{\alpha(w+\beta)^{2}} x^{-w} d w=\frac{1}{2}(\pi \alpha)^{-1 / 2} x^{\beta} e^{-(1 / 4 \alpha)(\ln x)^{2}}
$$

holds.

Proof. Let $f(x)=(1 / 2 \pi i) \int_{\mathcal{c}-i \infty}^{c+i \infty} e^{\alpha(w+\beta)^{2}} x^{-w} d w$ and $s=w+\beta$. Then $f(x)=\left(x^{\beta} / 2 \pi i\right) \int_{c^{*}-i \infty}^{c^{*}+i \infty} e^{\alpha s^{2}} x^{-s} d s$, where $c^{*}=c+\beta$. If $s=c^{*}+i z$, then $f(x)$ becomes

$$
\begin{aligned}
f(x)= & \frac{x^{\beta}}{2 \pi} \int_{-\infty}^{\infty} e^{\alpha\left(c^{*}+i z\right)^{2}} x^{-\left(c^{*}+i z\right)} d z \\
= & \frac{x^{\beta}}{2 \pi} \int_{-\infty}^{\infty} e^{-\alpha\left(z-i\left(c^{*}-\ln x / 2 \alpha\right)\right)^{2}} e^{-(1 / 4 \alpha)(\ln x)^{2}} d z \\
= & \frac{1}{2}(\pi \alpha)^{-1 / 2} x^{\beta} e^{-(1 / 4 \alpha)(\ln x)^{2}} \\
& \quad\left(\text { since } \int_{-\infty}^{\infty} e^{-z^{2}} d z=\sqrt{\pi}\right) .
\end{aligned}
$$

However, to apply Lemma 1 to (23), the following lemma is also required.

Lemma 2. For $M_{1}(\tau)$ and $G(\tau)$ given above, $(1 / 2) \sigma^{2} \tau+$ $M_{1}(\tau)+\eta(\tau-G(\tau)) \geq 0$ holds.

Proof. From the definition of $M_{1}(\tau)$ and $G(\tau)$,

$$
\begin{aligned}
\frac{1}{2} \sigma^{2} \tau & +M_{1}(\tau)+\eta(\tau-G(\tau)) \\
& =\frac{1}{2} \int_{0}^{\tau}\left(\sigma^{2}+2 \rho_{s r} \sigma \check{\sigma} G\left(t^{*}\right)+\check{\sigma}^{2} G^{2}\left(t^{*}\right)\right) d t^{*}
\end{aligned}
$$

is satisfied. However, since

$$
\begin{aligned}
0 & \leq\left(\sigma-\check{\sigma} G\left(t^{*}\right)\right)^{2} \leq \sigma^{2}+2 \rho_{s r} \sigma \check{\sigma} G\left(t^{*}\right)+\check{\sigma}^{2} G^{2}\left(t^{*}\right) \\
& \leq\left(\sigma+\check{\sigma} G\left(t^{*}\right)\right)^{2},
\end{aligned}
$$

we can prove $(1 / 2) \sigma^{2} \tau+M_{1}(\tau)+\eta(\tau-G(\tau)) \geq 0$, where $-1 \leq \rho_{s r} \leq 1$.
In (23), if we set

$$
\begin{gathered}
\alpha=\frac{1}{2} \sigma^{2} \tau+M_{1}(\tau)+\eta(\tau-G(\tau)), \\
\beta=\frac{1}{2}\left(1+\frac{M_{1}(\tau)-M_{2}(\tau)-G(\tau) r}{(1 / 2) \sigma^{2} \tau+M_{1}(\tau)+\eta(\tau-G(\tau))}\right),
\end{gathered}
$$

then, from Lemmas 1 and $2, \gamma(s)$ yields the following equation:

$\gamma(s)$

$$
\begin{aligned}
& =\exp \left\{-\frac{\left((1 / 2) \sigma^{2} \tau+\eta(\tau-G(\tau))+M_{2}(\tau)+G(\tau) r\right)^{2}}{4 \alpha}\right\} \\
& \quad \times \frac{1}{2 \sqrt{\alpha \pi}} s^{\beta} e^{-(1 / 4 \alpha)(\ln s)^{2}} .
\end{aligned}
$$

Now, we are trying to use relation to multiplicative convolution of Mellin transform and find $P_{n}(t, s, r)$. The Mellin convolution of $f$ and $g$ is given by the inverse Mellin transform of $\widehat{f}(w) \hat{g}(w)$ as follows:

$$
\begin{aligned}
f(x) * g(x) & =M_{w}^{-1}[\widehat{f}(w) \widehat{g}(w) ; x] \\
& =\int_{0}^{\infty} u^{-1} f\left(\frac{x}{u}\right) g(u) d u,
\end{aligned}
$$

where $f(x) * g(x)$ is the symbol of the Mellin convolution of $f$ and $g$ and $M_{w}^{-1}$ is the symbol of the inverse Mellin transform. It is referred to in [11] with more details.

In (21) and (22), since $e^{\varphi(\tau, w, r)}$ is the Mellin transform of $\gamma(s)$ and $E_{n}(w)$ is the Mellin transform of the payoff function $h_{n}(s)$, we have the following formula by using the Mellin convolution property mentioned above:

$$
\begin{aligned}
& P_{n}(\tau, s, r) \\
& =\int_{0}^{\infty} h_{n}(u) \gamma\left(\frac{s}{u}\right) \frac{1}{u} d u \\
& =\frac{1}{2 \sqrt{\alpha \pi}}\left[\int _ { K } ^ { n } ( u - K ) \operatorname { e x p } \left\{-\left(\frac{1}{2} \sigma^{2} \tau+\eta(\tau-G(\tau))\right.\right.\right. \\
& \left.\quad+M_{2}(\tau)+G(\tau) r\right)^{2} \\
& \left.\times(4 \alpha)^{-1}\right\} \\
& \left.\times\left(\frac{s}{u}\right)^{\beta} e^{-(1 / 4 \alpha)(\ln (s / u))^{2}} \frac{1}{u} d u\right] .
\end{aligned}
$$


Therefore, to find the European option price $P(t, s, r)$, if we take $n \rightarrow \infty$ in both sides of (31), then

$$
\begin{aligned}
P( & , s, r) \\
= & \lim _{n \rightarrow \infty} P_{n}(\tau, s, r) \\
= & \frac{1}{2 \sqrt{\alpha \pi}} \\
& \times\left[\int _ { K } ^ { \infty } ( u - K ) \operatorname { e x p } \left\{-\left(\frac{1}{2} \sigma^{2} \tau+\eta(\tau-G(\tau))\right.\right.\right. \\
= & \left.\left.\frac{1}{2 \sqrt{\alpha \pi}}+M_{2}(\tau)+G(\tau) r\right)^{2}(4 \alpha)^{-1}\right\} \\
& \times\left[\int_{K}^{\infty} u \exp \left\{-\left(\frac{s}{u}\right)^{\beta} e^{-(1 / 4 \alpha)(\ln (s / u))^{2}} \frac{1}{u} d u\right]\right. \\
& -\frac{1}{2 \sqrt{\alpha \pi}}\left[\int _ { K } ^ { \infty } K \operatorname { l e x p } \left\{-\left(\frac{1}{2} \sigma^{2} \tau+\eta(\tau-G(\tau))+M_{2}(\tau)\right.\right.\right. \\
& \left.\times\left(\frac{s}{u}\right)^{\beta} e^{-(1 / 4 \alpha)(\ln (s / u))^{2}} \frac{1}{u} d u\right] \\
\left.+G(\tau) r)^{2}(4 \alpha)^{-1}\right\} & +P_{2}(t, s, r) .
\end{aligned}
$$

Theorem 3. Under the payoff function $P(T, S, r)=\left(S_{T}-K\right)^{+}$, the formula of European call option with Hull-White interest rate is given by

$$
\begin{aligned}
P(t, s, r) & =P(T-\tau, s, r) \\
& =s \Phi\left(d_{1}\right)-K B(T-\tau, r ; T) \Phi\left(d_{2}\right), \\
d_{1}= & \frac{\ln (s / K)-\ln B(t, r ; T)+(1 / 2) \int_{0}^{\tau} \widehat{\sigma}^{2}\left(t^{*}\right) d t^{*}}{\sqrt{\int_{0}^{\tau} \hat{\sigma}^{2}\left(t^{*}\right) d t^{*}}}, \\
d_{2}= & d_{1}-\sqrt{\int_{0}^{\tau} \widehat{\sigma}^{2}\left(t^{*}\right) d t^{*}},
\end{aligned}
$$

where $B(T-\tau, r ; T)$ is the price of zero-coupon bond mentioned in Section 2.1, $\widehat{\sigma}(t)=\sqrt{\sigma^{2}+2 \rho_{s r} \sigma \check{\sigma} X(t)+\check{\sigma}^{2} X^{2}(t)}$ with $X(t)=-(1 / B)(\partial B / \partial r)=G(t)$, and $\Phi$ is the normal cumulative distribution function defined by $\Phi(z)=$ $(1 / \sqrt{2 \pi}) \int_{-\infty}^{z} e^{-z^{2} / 2} d z$.

Proof. Most of all, let $y=\ln (s / u) / \sqrt{2 \alpha}$. By applying change of variable $(u \rightarrow y), P_{1}(t, s, r)$ of (32) leads to

$$
\begin{aligned}
& P_{1}(t, s, r) \\
& =\frac{1}{2 \sqrt{\alpha \pi}}\left[\int _ { K } ^ { \infty } u \operatorname { e x p } \left\{-\left(\frac{1}{2} \sigma^{2} \tau+\eta(\tau-G(\tau))\right.\right.\right. \\
& \left.\left.+M_{2}(\tau)+G(\tau) r\right)^{2}(4 \alpha)^{-1}\right\} \\
& \left.\times\left(\frac{s}{u}\right)^{\beta} e^{-(1 / 4 \alpha)(\ln (s / u))^{2}} \frac{1}{u} d u\right] \\
& =\exp \left\{-\frac{\left((1 / 2) \sigma^{2} \tau+\eta(\tau-G(\tau))+M_{2}(\tau)+G(\tau) r\right)^{2}}{4 \alpha}\right\} \\
& \times \frac{1}{\sqrt{2 \pi}} s \int_{-\infty}^{\ln (s / K) / \sqrt{2 \alpha}} e^{\sqrt{2 \alpha}(\beta-1) y-(1 / 2) y^{2}} d y \\
& =\exp \left\{-\frac{\left((1 / 2) \sigma^{2} \tau+\eta(\tau-G(\tau))+M_{2}(\tau)+G(\tau) r\right)^{2}}{4 \alpha}\right. \\
& \left.+\alpha(\beta-1)^{2}\right\} \frac{1}{\sqrt{2 \pi}} s \\
& \times \int_{-\infty}^{\ln (s / K) / \sqrt{2 \alpha}} e^{-(1 / 2)(y-\sqrt{2 \alpha}(\beta-1))^{2}} d y \\
& =\exp \left\{-\frac{\left((1 / 2) \sigma^{2} \tau+\eta(\tau-G(\tau))+M_{2}(\tau)+G(\tau) r\right)^{2}}{4 \alpha}\right. \\
& \left.+\alpha(\beta-1)^{2}\right\} \frac{1}{\sqrt{2 \pi}} s \int_{-\infty}^{d_{1}} e^{-(1 / 2) z^{2}} d z
\end{aligned}
$$

where $d_{1}=(\ln (s / K)-2 \alpha(\beta-1)) / \sqrt{2 \alpha}$. From the definition of $\alpha, \beta$, and $B(T-\tau, r ; T)$ mentioned before, we have

$$
\begin{aligned}
& -\frac{1}{4} \frac{\left((1 / 2) \sigma^{2} \tau+\eta(\tau-G(\tau))+M_{2}(\tau)+G(\tau) r\right)^{2}}{\alpha} \\
& +\alpha(\beta-1)^{2}=0, \\
& 2 \alpha=\int_{0}^{\tau}\left(\sigma^{2}+2 \rho_{s r} \sigma \check{\sigma} G\left(t^{*}\right)+\check{\sigma}^{2} G^{2}\left(t^{*}\right)\right) d t^{*}, \\
& 2 \alpha(\beta-1)=\left(M_{1}(\tau)-M_{2}(\tau)-G(\tau) r\right) \\
& \quad-\left(\frac{1}{2} \sigma^{2} \tau+M_{1}(\tau)+\eta(\tau-G(\tau))\right) .
\end{aligned}
$$


Therefore, the solution of $P_{1}(t, s, r)$ is

$$
\begin{gathered}
P_{1}(t, s, r)=s \frac{1}{\sqrt{2 \pi}} \int_{-\infty}^{d_{1}} e^{-(1 / 2) z^{2}} d z, \\
d_{1}=\frac{\ln (s / K)-\ln B(t, r ; T)+(1 / 2) \int_{0}^{\tau} \widehat{\sigma}^{2}\left(t^{*}\right) d t^{*}}{\sqrt{\int_{0}^{\tau} \widehat{\sigma}^{2}\left(t^{*}\right) d t^{*}}},
\end{gathered}
$$

where $\widehat{\sigma}^{2}(t)=\sigma^{2}+2 \rho_{s r} \sigma \check{\sigma} G(t)+\check{\sigma}^{2} G^{2}(t)$.

Also, by setting $y=\ln (s / u) / \sqrt{2 \alpha}$ and using the similar way stated above, $P_{2}(t, s, r)$ of (32) is transformed into

$$
\begin{aligned}
& P_{2}(t, s, r) \\
& =\frac{1}{2 \sqrt{\alpha \pi}}\left[\int _ { K } ^ { \infty } K \operatorname { e x p } \left\{-\left(\frac{1}{2} \sigma^{2} \tau+\eta(\tau-G(\tau))\right.\right.\right. \\
& \left.\left.+M_{2}(\tau)+G(\tau) r\right)^{2}(4 \alpha)^{-1}\right\} \\
& \left.\times\left(\frac{s}{u}\right)^{\beta} e^{-(1 / 4 \alpha)(\ln (s / u))^{2}} \frac{1}{u} d u\right] \\
& =\exp \left\{-\frac{\left((1 / 2) \sigma^{2} \tau+\eta(\tau-G(\tau))+M_{2}(\tau)+G(\tau) r\right)^{2}}{4 \alpha}\right. \\
& \left.+\alpha \beta^{2}\right\} \frac{1}{\sqrt{2 \pi}} K \int_{-\infty}^{\ln (s / K) / \sqrt{2 \alpha}} e^{-(1 / 2)(y-\sqrt{2 \alpha} \beta)^{2}} d y \\
& =\exp \left\{-\frac{\left((1 / 2) \sigma^{2} \tau+\eta(\tau-G(\tau))+M_{2}(\tau)+G(\tau) r\right)^{2}}{4 \alpha}\right. \\
& \left.+\alpha \beta^{2}\right\} \frac{1}{\sqrt{2 \pi}} K \int_{-\infty}^{\ln (s / K) / \sqrt{2 \alpha}-\sqrt{2 \alpha} \beta} e^{-(1 / 2) z^{2}} d z .
\end{aligned}
$$

Similarly,

$$
\begin{aligned}
& \exp \left\{-\frac{\left((1 / 2) \sigma^{2} \tau+\eta(\tau-G(\tau))+M_{2}(\tau)+G(\tau) r\right)^{2}}{4 \alpha}\right. \\
& \left.\quad+\alpha \beta^{2}\right\} \\
& \quad=\exp \left\{M_{1}(\tau)-M_{2}(\tau)-G(\tau) r\right\}=B(T-\tau, r ; T), \\
& \frac{\ln (s / K)}{\sqrt{2 \alpha}}-\sqrt{2 \alpha} \beta \\
& =\frac{\ln (s / K)-\ln B(t, r ; T)-(1 / 2) \int_{0}^{\tau} \widehat{\sigma}^{2}\left(t^{*}\right) d t^{*}}{\sqrt{\int_{0}^{\tau} \widehat{\sigma}^{2}\left(t^{*}\right) d t^{*}}} .
\end{aligned}
$$

Therefore, the solution of $P_{2}(t, s, r)$ is

$$
\begin{gathered}
P_{2}(t, s, r)=K B(T-\tau, r ; T) \frac{1}{\sqrt{2 \pi}} \int_{-\infty}^{d_{2}} e^{-(1 / 2) z^{2}} d z, \\
d_{2}=\frac{\ln (s / K)-\ln B(t, r ; T)-(1 / 2) \int_{0}^{\tau} \widehat{\sigma}^{2}\left(t^{*}\right) d t^{*}}{\sqrt{\int_{0}^{\tau} \widehat{\sigma}^{2}\left(t^{*}\right) d t^{*}}} .
\end{gathered}
$$

Finally, by combining (32), (36), and (39), we obtain the following result:

$$
\begin{aligned}
P(t, s, r)= & P(T-\tau, s, r) \\
= & s \int_{-\infty}^{d_{1}} e^{-(1 / 2) z^{2}} d z-K B(T-\tau, r ; T) \\
& \times \int_{-\infty}^{d_{2}} e^{-(1 / 2) z^{2}} d z, \\
d_{1}= & \frac{\ln (s / K)-\ln B(t, r ; T)+(1 / 2) \int_{0}^{\tau} \widehat{\sigma}^{2}\left(t^{*}\right) d t^{*}}{\sqrt{\int_{0}^{\tau} \widehat{\sigma}^{2}\left(t^{*}\right) d t^{*}}}, \\
d_{2}= & d_{1}-\sqrt{\int_{0}^{\tau} \widehat{\sigma}^{2}\left(t^{*}\right) d t^{*} .}
\end{aligned}
$$

The proof is completed.

\subsection{The Case of Put Option}

Corollary 4. If $P^{*}(T, S, r)=\left(K-S_{T}\right)^{+}$is European vanilla put option with Hull-White interest rate, then the formula leads to the following relation:

$$
\begin{aligned}
P^{*}(t, s, r) & =P^{*}(T-\tau, s, r) \\
& =K B(T-\tau, r ; T) \Phi\left(-d_{2}\right)-s \Phi\left(-d_{1}\right), \\
d_{1}= & \frac{\ln (s / K)-\ln B(t, r ; T)+(1 / 2) \int_{0}^{\tau} \widehat{\sigma}^{2}\left(t^{*}\right) d t^{*}}{\sqrt{\int_{0}^{\tau} \widehat{\sigma}^{2}\left(t^{*}\right) d t^{*}}}, \\
d_{2}= & d_{1}-\sqrt{\int_{0}^{\tau} \widehat{\sigma}^{2}\left(t^{*}\right) d t^{*}} .
\end{aligned}
$$

Proof. In case of European put option, one has the payoff function $P^{*}(T, S, r)=h^{*}(S)=\left(K-S_{T}\right)^{+}$. Unlike the case of call option, for the payoff of the put option, the Mellin transform $\widehat{h}(w)=\int_{0}^{\infty} h(s) s^{w-1} d w$ exists and the value is given by $\widehat{h}^{*}(w)=K^{w+1} / w(w+1)$. Therefore, for the function $\widehat{h}^{*}(w)$, we can apply the same Mellin transform method used to derive the formula of call option and then we obtain the formula of European vanilla put option with Hull-White interest rate stated above.

\section{Concluding Remarks}

In this paper, we have investigated that a closed formula for the European option price under Hull-White interest rate 
can be derived by applying the method of Mellin transforms. The formula is simply given by the normal cumulative distribution function. Also, one can apply the Mellin transform methods to other types of options except the ones that we did already.

\section{Conflict of Interests}

The author declares that there is no conflict of interests regarding the publication of this paper.

\section{Acknowledgments}

The author would like to thank the referees for their valuable comments and suggestions to improve the paper. The research of Ji-Hun Yoon was supported by BK21 PLUS SNU Mathematical Sciences Division.

\section{References}

[1] R. C. Merton, “Theory of rational option pricing," The Rand Journal of Economics, vol. 4, pp. 141-183, 1973.

[2] R. Rabinovitch, "Pricing stock and bond options when the default-free rate is stochastic," Journal of Financial and Quantitative Analysis, vol. 24, pp. 447-457, 1989.

[3] K. I. Amin and R. A. Jarrow, "Pricing options on risky assets in a stochastic interest rate economy," Mathematical Finance, vol. 4, pp. 217-237, 1992.

[4] Y.-J. Kim, "Option pricing under stochastic interest rates: an empirical investigation," Asia-Pacific Financial Markets, vol. 9, no. 1, pp. 23-44, 2002.

[5] H. Fang, "European option pricing formula under stochastic interest rate," Progress in Applied Mathematics, vol. 4, no. 1, pp. 14-21, 2012.

[6] R. Panini and R. P. Srivastav, "Option pricing with Mellin transforms," Mathematical and Computer Modelling, vol. 40, no. 1-2, pp. 43-56, 2004.

[7] R. Panini and R. P. Srivastav, "Pricing perpetual options using Mellin transforms," Applied Mathematics Letters, vol. 18, no. 4, pp. 471-474, 2005.

[8] R. Frontczak and R. Schöbel, "On modified Mellin transforms, Gauss-Laguerre quadrature, and the valuation of American call options," Journal of Computational and Applied Mathematics, vol. 234, no. 5, pp. 1559-1571, 2010.

[9] Z. A. Elshegmani and R. R. Ahmed, "Analytical solution for an arithmetic Asian option using Mellin transforms," International Journal of Mathematical Analysis, vol. 5, no. 26, pp. 1259-1265, 2011.

[10] B. Øksendal, Stochastic Differential Equations, Springer, New York, NY, USA, 2003.

[11] J. Bertrand, P. Bertrand, and J. Ovarlez, "The mellin transform," in The Transforms and Applications Handbook, D. Alexander, Ed., CRC Press, Boca Raton, Fla, USA, 2nd edition, 2000. 


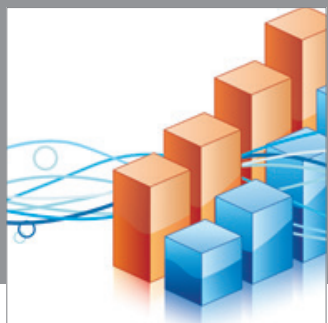

Advances in

Operations Research

mansans

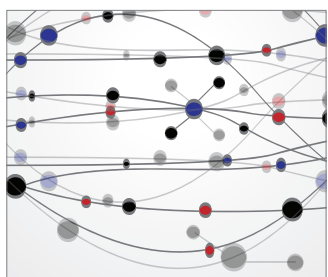

The Scientific World Journal
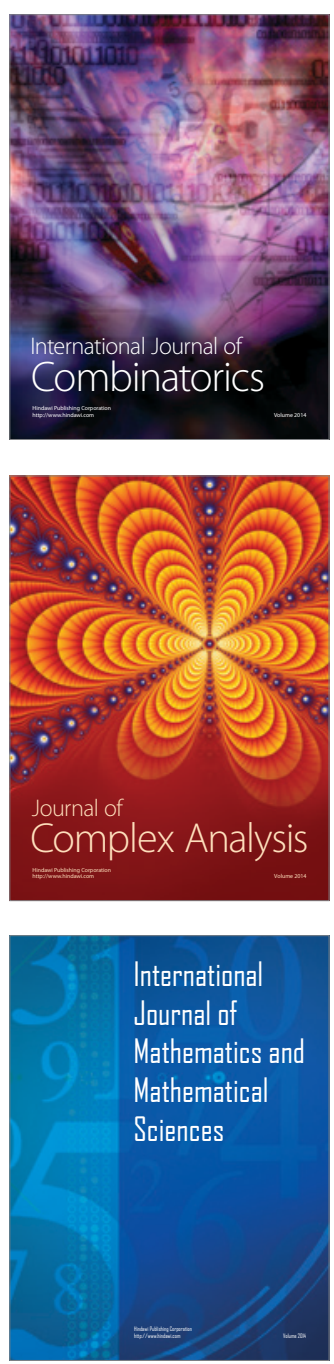
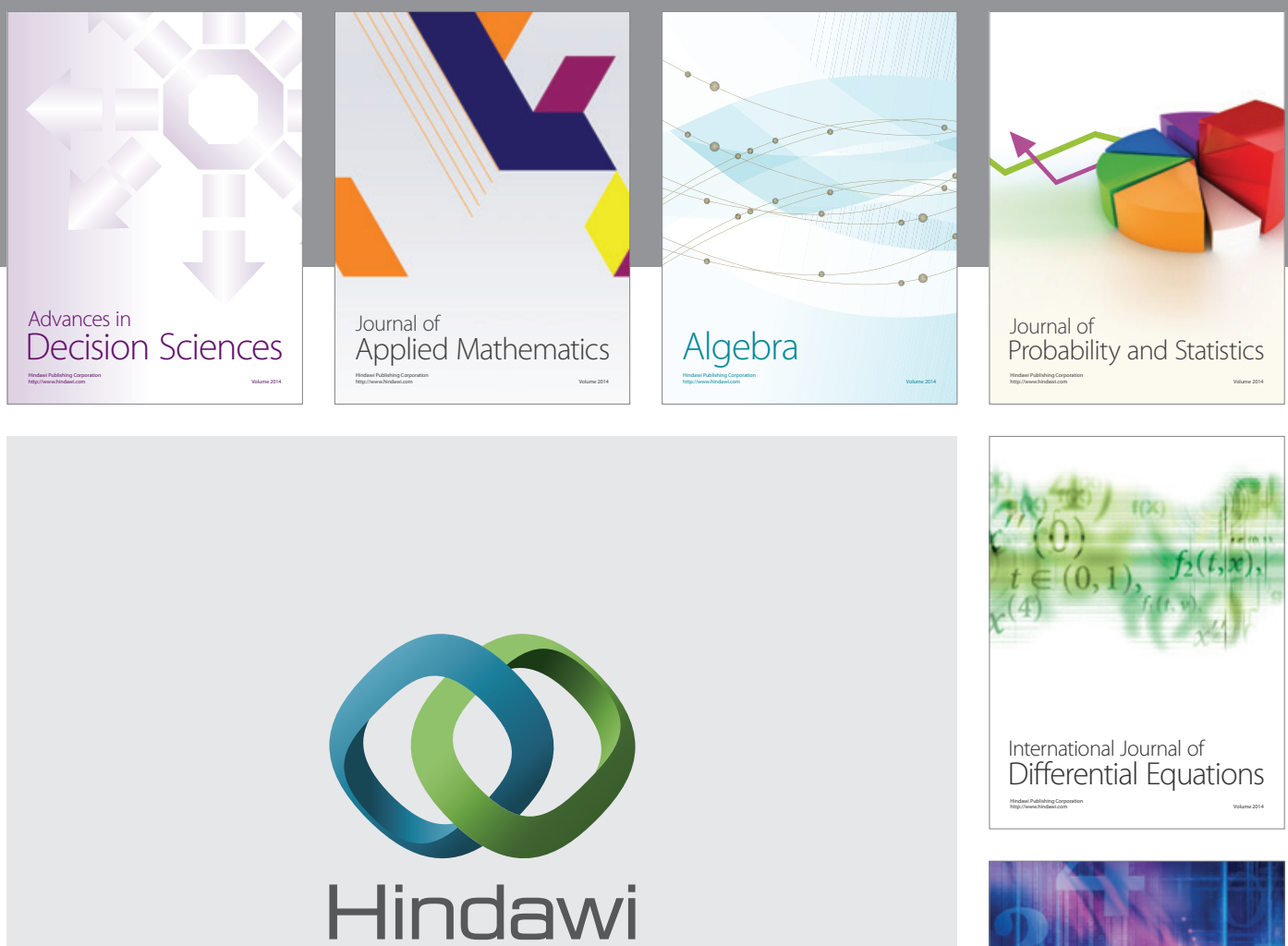

Submit your manuscripts at http://www.hindawi.com
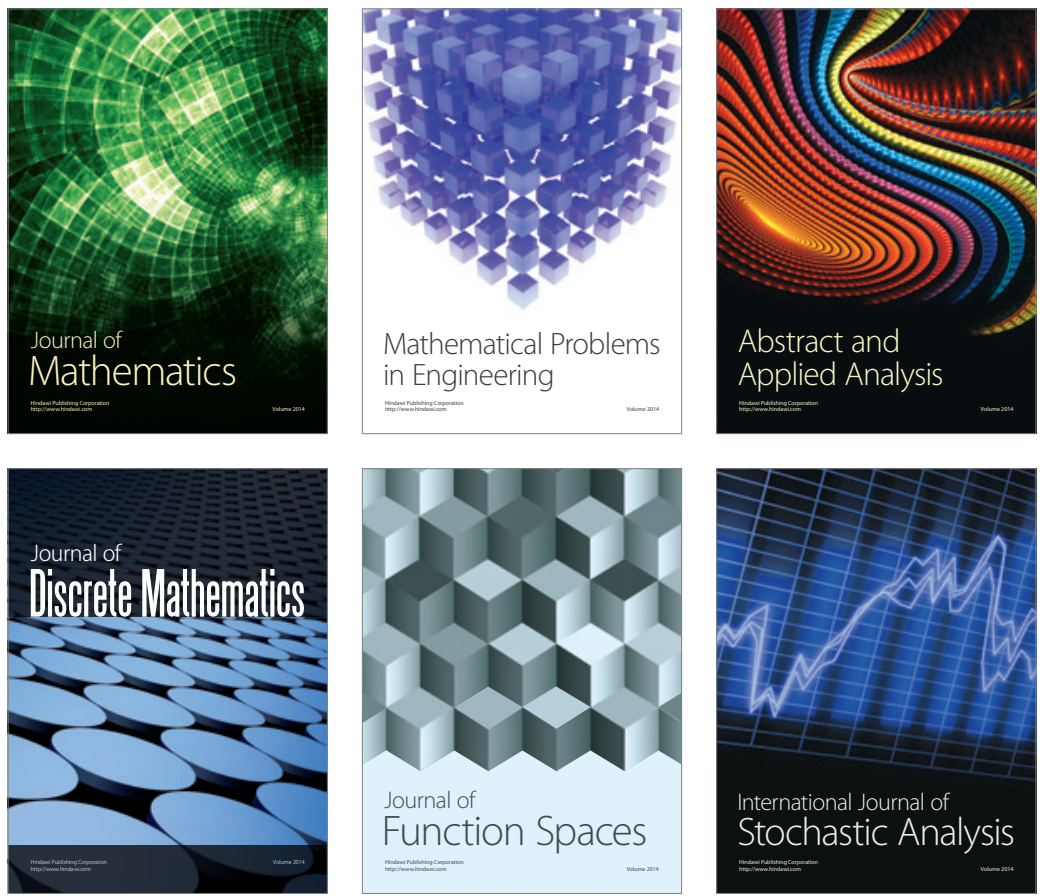

Journal of

Function Spaces

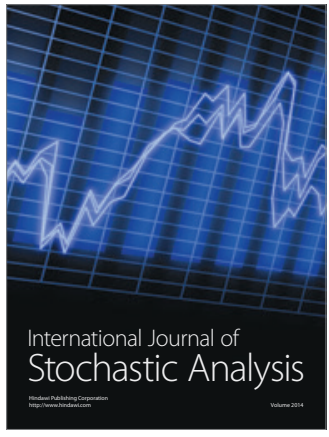

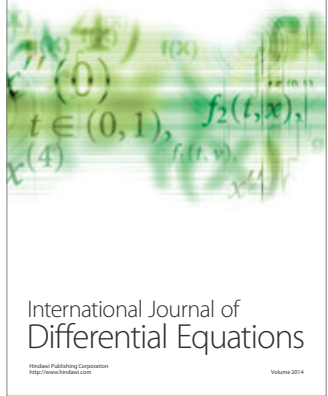
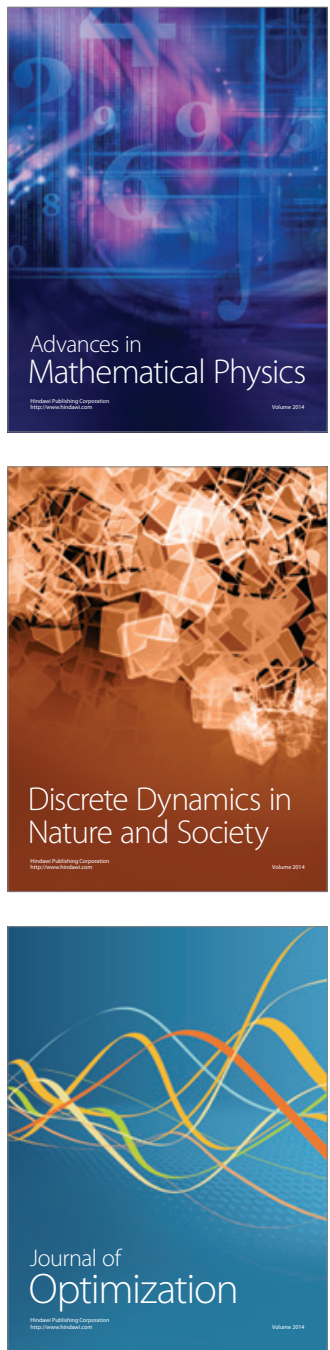